\title{
Measurement-Free Topological Protection Using Dissipative Feedback
}

\author{
Keisuke Fujii, ${ }^{1,2,3}$ Makoto Negoro, ${ }^{1}$ Nobuyuki Imoto, ${ }^{1}$ and Masahiro Kitagawa ${ }^{1}$ \\ ${ }^{1}$ Graduate School of Engineering Science, Osaka University, \\ 1-3 Machikaneyama, Toyonaka, Osaka 560-8531, Japan \\ ${ }^{2}$ The Hakubi Center for Advanced Research, Kyoto University, \\ Yoshida-Ushinomiya-cho, Sakyo-ku, Kyoto 606-8302, Japan \\ ${ }^{3}$ Graduate School of Informatics, Kyoto University, Yoshida Honmachi, Sakyo-ku, Kyoto 606-8501, Japan
}

(Received 12 June 2014; revised manuscript received 3 October 2014; published 1 December 2014)

\begin{abstract}
Protecting quantum information from decoherence due to environmental noise is vital for fault-tolerant quantum computation. To this end, standard quantum error correction employs parallel projective measurements of individual particles, which makes the system extremely complicated. Here, we propose measurement-free topological protection in two dimensions without any selective addressing of individual particles. We make use of engineered dissipative dynamics and feedback operations to reduce the entropy generated by decoherence in such a way that quantum information is topologically protected. We calculate an error threshold, below which quantum information is protected, without assuming selective addressing, projective measurements, or instantaneous classical processing. All physical operations are local and translationally invariant, and no parallel projective measurement is required, which implies high scalability. Furthermore, since the engineered dissipative dynamics we utilize has been well studied in quantum simulation, the proposed scheme can be a promising route progressing from quantum simulation to fault-tolerant quantum information processing.
\end{abstract}

DOI: 10.1103/PhysRevX.4.041039

\section{INTRODUCTION}

A standard approach to protect quantum information from decoherence is the use of the celebrated quantum error correction (QEC) [1-3]. It conventionally employs projective measurements, classical information processing, and feedback operations with selective addressing of individual particles. Based on the standard paradigm of QEC, fault-tolerant quantum computer architectures have been designed with the additional ability to operate universal quantum gates [4-14].

However, there exist severe problems that have to be overcome for the realization of standard QEC. The projective measurements and classical processing utilized in standard QEC have to be much faster than the coherence time of quantum systems, which is extremely challenging in experiments. If classical processing depends on the size of the system, it ultimately limits both the speed and the size of quantum computers. From a theoretical viewpoint, it is highly nontrivial to establish an error threshold theory including the classical system to control quantum computers. In practice, fast and reliable parallel projective measurements of the massive numbers of qubits employed

Published by the American Physical Society under the terms of the Creative Commons Attribution 3.0 License. Further distribution of this work must maintain attribution to the author(s) and the published article's title, journal citation, and DOI.
Subject Areas: Condensed Matter Physics,

Quantum Physics, Quantum Information

in conventional QEC are very challenging (see Fig. 1). A recent study [15] has revealed that parallel projective measurements of individual $10^{8}$ qubits are required every hundred nanoseconds to maintain quantum coherence for factorization of 1024-bit composite numbers. (The total amount of information measured is 1 petabit/ sec.) While monolithic architectures, quantum dots [15], and superconducting qubits $[16,17]$ on a chip exhibit promising scalability, macroscopic measurement devices coupled with individual qubits for parallel projective measurements might introduce other sources of decoherence, thereby limiting this approach. On the other hand, distributed architectures, consisting of modules comprising a small number of qubits, connected with optical channels, allow both accurate manipulations and measurements inside the local modules [18-25]. However, the entangling operation between separate local modules using flying photons takes a long time due to photon loss. Hopefully, these problems will be overcome within the conventional paradigm by a breakthrough in the development of accurate manipulations and parallel projective measurement technology. In the meantime, we should not stop searching for a novel way toward robust and scalable protection of quantum information.

Here, we propose a new paradigm, measurement-free topological protection (MFTP) of quantum information using dissipative dynamics, paving a novel way toward fault-tolerant quantum computation. We unify the quantum system that is to be protected and a controlling classical 

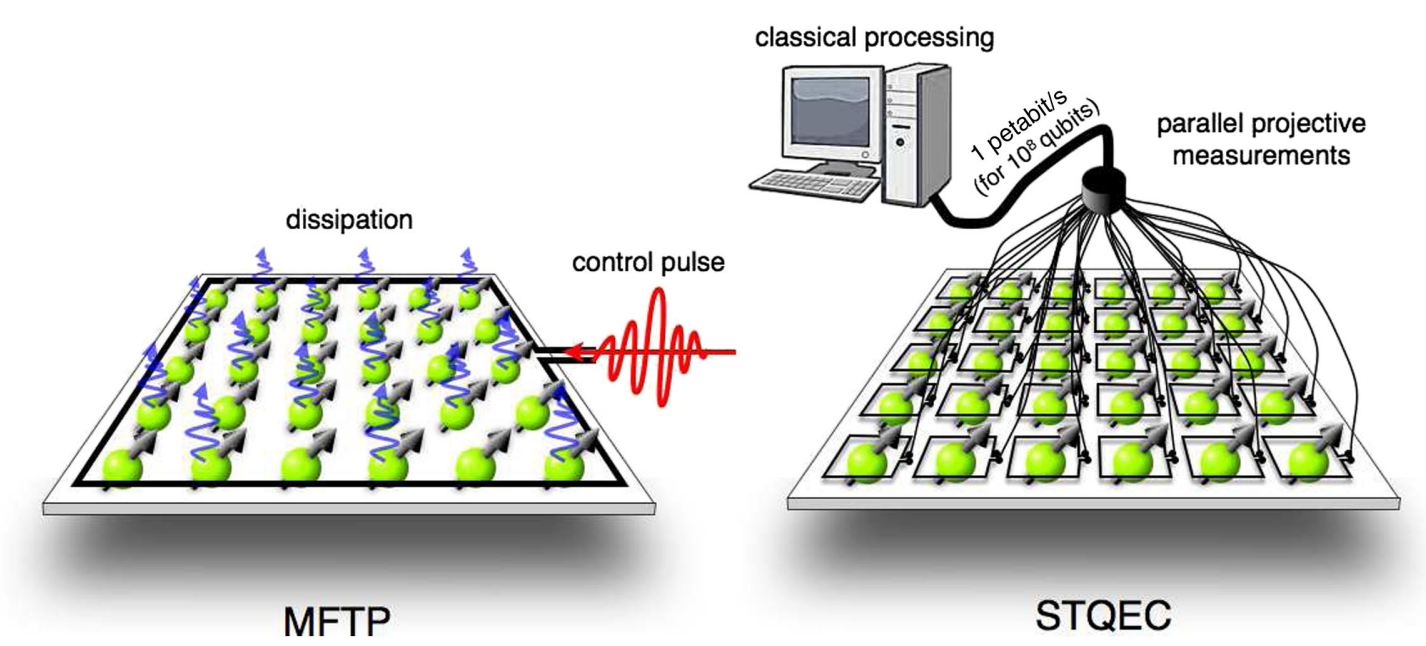

FIG. 1. MFTP versus standard topological QEC (STQEC). MFTP with discrete-dissipative feedback operations, which are implemented by local and translationally invariant physical operations (left). A STQEC with projective measurements and classical processing (right). Since MFTP does not require any selective addressing, its scalability is evident.

system in a framework without assuming parallel projective measurements, selective addressing, or instantaneous classical processing. Specifically, we devise a way to correct errors on topological quantum codes with restricting our controllability to translationally invariant nearestneighbor unitary operations and single-spin dissipative dynamics in a two-dimensional system as follows. The classical system is coupled with the quantum system, in which quantum information is stored, in order to extract the syndrome of errors. With this information, the classical system is cooled down by an engineered dissipative dynamics. (See Ref. [3] for a comprehensive review of quantum error correction and an engineered dissipation aimed at quantum memory.) The obtained low-energy state in the classical system is utilized with a feedback operation to correct errors on the quantum information in the quantum system. The lifetime of the stored quantum information is shown to be improved polynomially in the size (linear length) of the system, provided that the error probability is smaller than a threshold value.

Since the proposed topological protection does not require any selective addressing or parallel projective measurements, MFTP enables us to easily achieve scalability in various physical systems (see Fig. 1). Furthermore, engineered dissipative dynamics utilized for topological protection has been well studied in the context of quantum simulation [26-29]. MFTP serves as a promising route to progress from quantum simulation to fault-tolerant quantum information processing.

\section{TOPOLOGICAL PROTECTION}

We consider a 2D many-body quantum system that encodes quantum information, for simplicity, using the surface code [30,31] [the lower layer in Fig. 2(a)]. The proposed scheme can also be applied straightforwardly to other local stabilizer codes such as topological color codes
[32]. The surface code, in which a qubit is located on each edge of an $L \times L$ square lattice as shown in Fig. 2(a), is stabilized by the face and vertex stabilizer operators, $A_{f}=\prod_{i \in E_{f}} Z_{i}$ and $B_{v}=\prod_{j \in E_{v}} X_{j}$, respectively. Here, $Z_{i}$ and $X_{j}$ denote Pauli operators on the $i$ th and $j$ th qubits, and $E_{f}$ and $E_{v}$ indicate the sets of four edges surrounding a face $f$ and adjacent to a vertex $v$, respectively. The error syndromes $\left\{a_{f}\right\}$ and $\left\{b_{v}\right\}$ are defined as sets of eigenvalues of the face and vertex stabilizers, respectively, which are used to identify $X$ and $Z$ errors. These errors are assumed to occur on each qubit with independent and identical error probability $p$, for simplicity.

In standard topological quantum error correction (STQEC) [3,31], the error syndrome is extracted to the classical world through parallel projective measurements. According to the error syndrome, we use a classical algorithm, minimum-weight perfect matching (MWPM) [33], which tells us the location of the errors. By virtue of the locality and translational invariance of the surface code, the error threshold of the STQEC is very high $(\sim 1 \%)$ even when using only the nearest-neighbor two-qubit gates to extract the error syndrome [10-12]. However, the necessity of parallel projective measurements and the classical processing may limit the effectiveness of STQEC.

If measurement-free QEC is allowed, both of these limiting factors can be eliminated in fault-tolerant quantum computation. In Refs. [34,35], measurement-free QEC with a nontopological code was investigated, where errors are corrected by unitary dynamics with selective addressability of the boundary qubits. It is well known that the projective measurements can be replaced with preparations of fresh ancillas (by dissipation) followed by controlled-unitary operations. However, if we straightforwardly apply this strategy to the surface code, the classical processing, i.e., MWPM, is too complex to be implemented by unitary dynamics. The unitary dynamics for MWPM is far from 

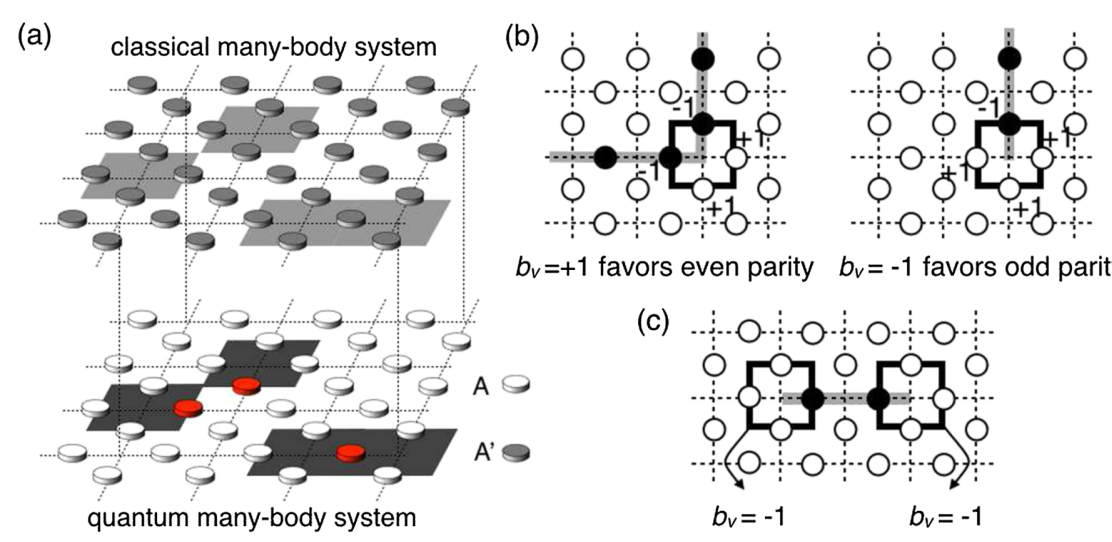

$b_{v}=+1$ favors even parity $\quad b_{v}=-1$ favors odd parity

(c)

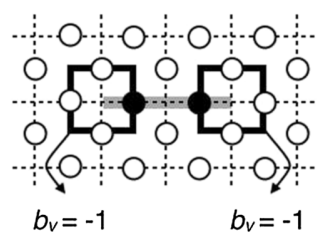

FIG. 2. The system and dissipative dynamics for MFTP. (a) The quantum (lower layer) and classical (upper layer) many-body systems for quantum information storage and its feedback controls, respectively. The $Z$ errors occur on the qubits colored by red. The associated vertex stabilizers with the eigenvalue -1 are denoted by black squares. The four classical spins adjacent to each vertex interact according to the eigenvalues of the vertex stabilizers. The wrong-sign plaquettes with $b_{v}=-1$ are shown by gray squares on the upper layer. The double layer is not necessarily required, but different degrees of freedom of the particles located on one layer can be employed. (b) The effect of plaquette interaction. A correct-sign plaquette with $b_{v}=+1$ favors an even number of -1 's, where a chain of -1 's is energetically hard to terminate. The wrong-sign plaquette favors an odd number of -1 's, where a chain of -1 's terminates easily. (c) The competing interactions reveal the location of errors: two wrong-sing plaquettes are connected with a short length.

local and translationally invariant, which completely diminishes the merit of using the surface code. This is also the case for topological quantum error correction by a sophisticated local update rule, which requires a complex classical ancilla system [36]. It is nontrivial to design the system to be topologically protected with local and translationally invariant physical operations, which are feasible enough to implemented in an actual experiment. The proposed scheme, MFTP, makes active use of dissipative dynamics to reduce the entropy of the quantum system in such a way that quantum information is topologically protected with local and translationally invariant operations, thus enabling us to fully utilize the advantage of the surface code.

\section{DISCRETE-DISSIPATIVE FEEDBACK FOR TOPOLOGICAL PROTECTION}

Recently, extensive research has been conducted on engineering dissipative dynamics to simulate open quantum systems [26] or to prepare quantum states of interest [37-40]. Unfortunately, the local and translationally invariant dissipations toward thermal equilibrium at finite temperature cannot protect quantum information due to no-go theorems of self-correcting quantum memory [41,42]. While extensive effort is being made, such as the toricboson model [43], a self-correcting quantum memory has not been achieved by dissipative dynamics under a fixed local Hamiltonian system. A quantum version of a nonvolatile magnetic storage device in equilibrium, such as a hard disk drive, seems to be hard to achieve at finite temperature. Not only dissipative dynamics but also the time dependence of the Hamiltonian, which drives the system into nonequilibrium with a feedback mechanism, would be key ingredients to achieve topological protection.
In this sense, the proposed model achieving topological protection of quantum information in nonequilibrium can be regarded as a quantum analog of dynamic memory, which periodically refreshes the capacitor charge to store information reliably.

We utilize a classical 2D many-body system, in addition to the quantum system, as an ancilla to allow feedback operations of discrete type [the upper layer in Fig. 2(a)]. For simplicity, we explain the feedback operation for the $Z$ error correction only. (The $X$ error correction can also be done in a similar way.) According to the error syndrome $\left\{b_{v}\right\}$, we cool (dissipate the energy) the classical system down to a sufficiently low but a finite temperature $T$ under a Hamiltonian

$$
H\left(\left\{b_{v}\right\}\right)=-J \sum_{v} b_{v} \prod_{i \in E_{v}} u_{i}-h \sum_{i} u_{i}
$$

where $u_{i}= \pm 1$ denotes the classical spin located on edge $i$ and $J$ and $h$ indicate the coupling constant and the magnetic field strength, respectively. Here the "classical" spins refers to the qubits without their phase coherence. We call this Hamiltonian the 2D random-plaquette gauge model with magnetic fields. Then the equilibrium configuration is obtained and put back into the quantum system by transversal controlled- $Z$ (CZ) operations between the classical (controls) and quantum (targets) systems.

The above discrete-dissipative feedback process succeeds in correcting errors in the surface code through the following progression. The location of errors in the surface code can be identified by finding a minimum path connecting pairs of incorrect eigenvalues $b_{v}=-1$ [31]. The first term in Eq. (1), which we call the plaquette 

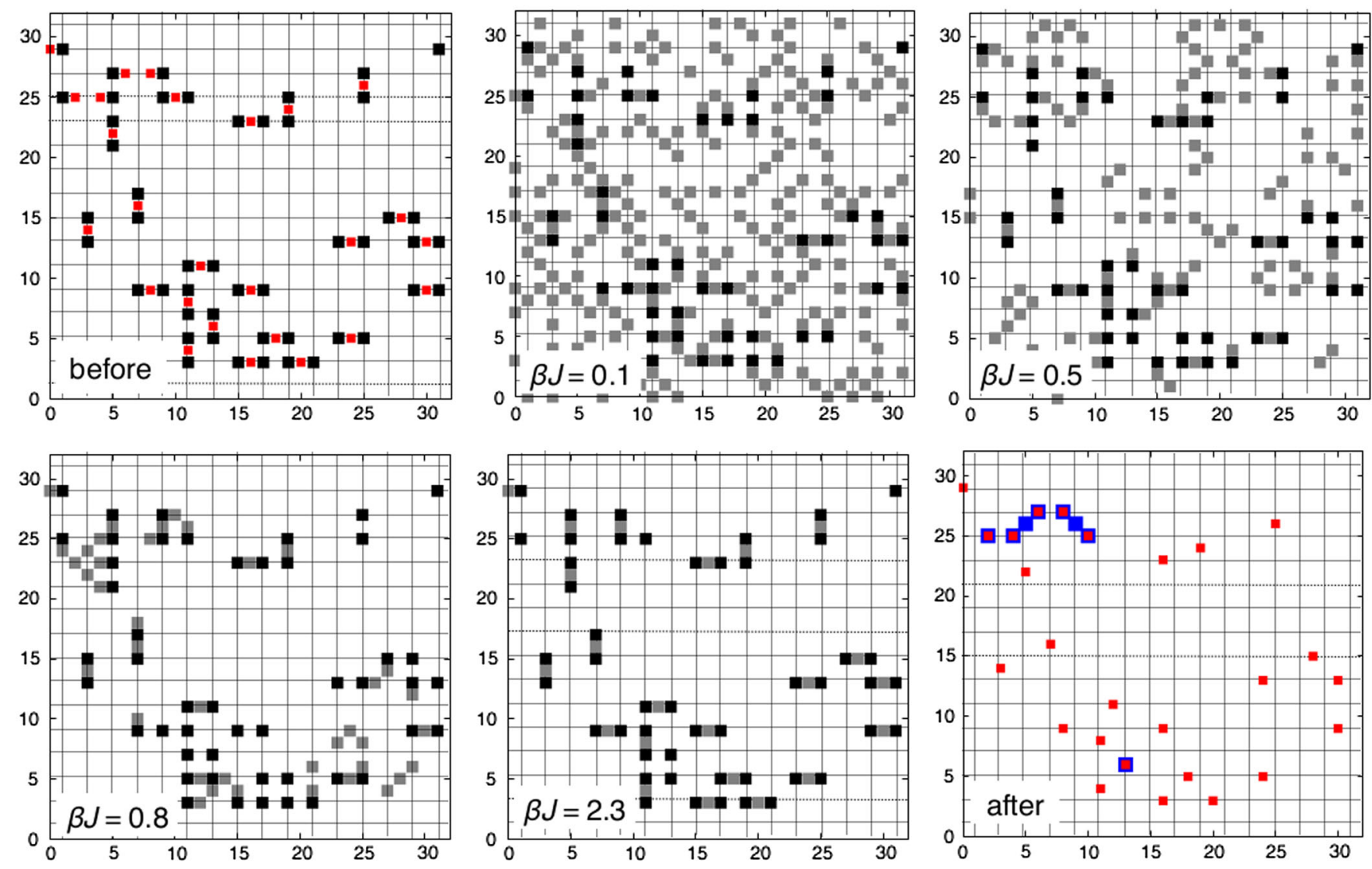

FIG. 3. Cooling dynamics and topological error correction. The location of the $Z$ errors in the quantum system and wrong-sign plaquettes are shown by red and black squares, respectively (top left), where the error probability is $p=0.05$. Equilibrium configurations are shown for $\beta J=0.1$ (top middle), 0.5 (top right), 0.8 (bottom left), and 2.3 (bottom middle), where -1 spins are shown by gray squares. An equilibrium configuration at low temperature is put back into the quantum system. The remaining $Z$ errors after the feedback are shown by blue squares (bottom right). Most of the errors are corrected, but some errors, shown by blue squares, remain. These are corrected by subsequent cycles.

interactions, imposes the boundary conditions of a chain of -1 's as shown in Fig. 2(b). A correct-sign plaquette with $b_{v}=1$ energetically favors an even number of -1 's, where a chain of -1 's is hard to terminate. A wrong-sign plaquette with $b_{v}=-1$ energetically favors an odd number of -1 's, where a chain of -1 's terminates easily. On the other hand, the second term in Eq. (1), which we call magnetic fields, favors configurations of fewer $-1 \mathrm{~s}$ '. If the plaquette interactions are chosen to be strong enough to connect pairs of wrong-sign plaquettes in the presence of the magnetic fields, these two competing interactions are expected to reveal the locations of the $Z$ errors at a sufficiently low temperature [see Fig. 2(c)]. In Appendix A, we show that, by choosing the strength of plaquette interactions such that $4 J /(2 h)=\alpha \log L$ with a constant $\alpha$, the logical error probability per step decreases polynomially in the system size $L$, as long as the physical error probability $p$ is below a threshold value. Since the correlation length of the system scales like $O(\log L)$, the relaxation time is expected to depend on the system size $L$ polylogarithmically (see Appendix A).
In order to confirm the above observation, we perform numerical simulations of the classical cooling process under the Hamiltonian $H\left(\left\{b_{v}\right\}\right)$ using the Metropolis method. Figure 3 illustrates equilibrium configurations at $\beta h=0.1,0.5,0.8$, and 2.3 , where $\beta$ is the inverse temperature and $J=h$ is adopted, for example. The remaining $Z$ errors after the feedback operation are also shown. Most of the errors are corrected within one cycle of MFTP, as shown in Fig. 3 (lower right-hand panel). However, some errors still remain, which are attributed to the excitations in the plaquette interactions that result from a finite temperature effect. Such errors are corrected in the following MFTP cycles and/or suppressed by the logarithmic scaling of the plaquette interactions $4 J /(2 h)=\alpha \log L$.

We further investigate the logical error probability with varying the system size $L$ and the physical error probability $p$. Specifically, we choose $\alpha=1$ and utilize the thermal equilibrium state of a temperature $T=1 / \beta=-2 h / \ln [p /(1-p)]$ on the Nishimori line [44], where the thermal fluctuation and physical error probability are balanced. In the limit of large $J$ (i.e., large $\alpha$ ), the discrete-dissipative feedback under the 

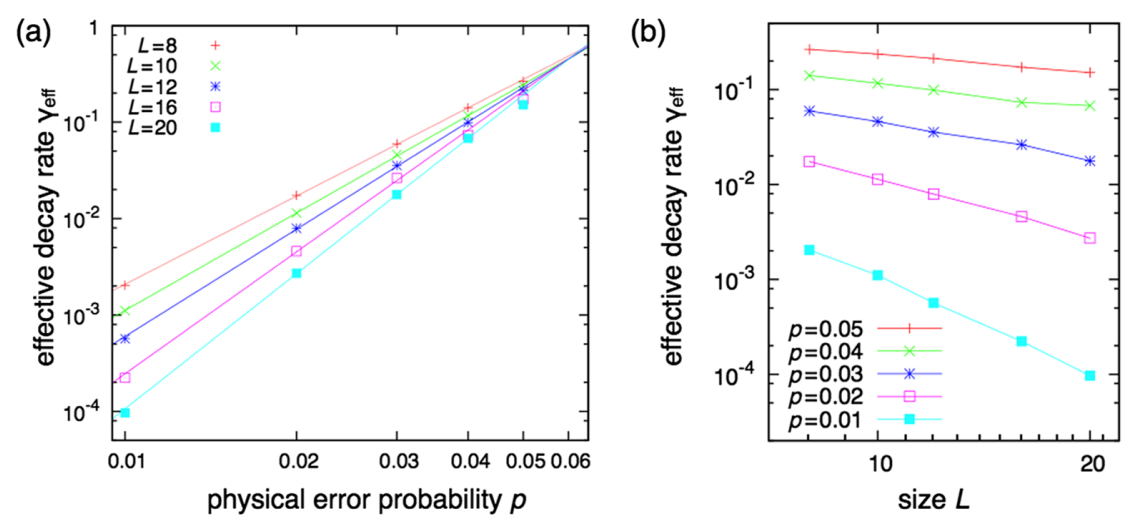

FIG. 4. Numerical results. The effective decay rate $\gamma_{\text {eff }}$ is estimated by numerical simulations for the physical error probabilities $p=0.01,0.02, \ldots, 0.05$ and the system size $L=8,10,12,16,20$. (a) The effective decay rate $\gamma_{\text {eff }}$ as a function of the physical error probability $p$. From top to bottom $L=8,10,12,16,20$. (b) The effective decay rate $\gamma_{\text {eff }}$ as a function of the system size $L$. From bottom to top $p=0.01,0.02,0.03,0.04,0.05$.

Nishimori temperature achieves an optimal decoding of the surface code. The effective decay rate $\gamma_{\text {eff }}$ of the stored information under topological protection is calculated by fitting the logical error probability to $3\left(1-e^{-\gamma_{\text {eff }} t}\right) / 4$ with $t$ being the time step. Since two logical qubits are encoded into the surface code, the fitting is chosen such that the logical error probability converges to $3 / 4$ in the large $t$ limit. (The fidelity of a maximally mixed state of two qubits is $1 / 4$.) The effective decay rate $\gamma_{\text {eff }}$ is plotted as functions of the physical error probability $p$ and the size $L$ in Figs. 4(a) and 4(b), respectively. We further analyze the scaling of the effective decay rate with respect to the linear length $L$ of the system and the physical error probability $p$. We obtain $\Gamma_{\text {eff }}=D L^{[E \log (p)+F]}$ with $D=0.82-1.15$, $E=1.54 \pm 0.05$, and $F=4.06 \pm 0.18$. This indicates that the lifetime of the quantum information is improved polynomially in the size of the system $L$, which is in good agreement with the theoretical analysis made in Appendix A. We observe that the threshold for physical error probability with $\alpha=1$ is at least as high as $5.0 \%$. The extrapolation of Fig. 4 (a) or equivalently the condition $E \log (p)+F<0$ provides the threshold value around $6.0 \%-7.0 \%$.

\section{IMPLEMENTATION AND FEASIBILITY OF MFTP}

We now consider a physical implementation of MFTP. We consider a system with two layers, each of which consists of three species of particles, as shown in Fig. 5(a) (left-hand side). The A qubits and A' spins are the quantum and classical systems considered, respectively. The B and C qubits are the ancillas for the $Z$ and $X$ error syndrome extractions, respectively. The B' and C' spins are used to mediate the syndrome-dependent plaquette interactions. Instead of the double-layer system, particles on a single layer, which have different degrees of freedom, can be utilized as shown in Fig. 5(a) (right-hand side).
We utilize simultaneous two-qubit gates on neighboring particles of different species, such as controlled-NOT (СNOT) gates between A and B qubits, for example. In addition, we use a dissipative operation, specifically $T_{1}$ relaxation or incoherent pumping, on the classical spins. The procedure in each MFTP cycle for the $Z$ error correction is as follows (see also Fig. 6). (i) Initialize B and B' to $|0\rangle$. (ii) Perform CNOT gate operations between A (controls) and B (targets) to extract the error syndrome. Perform CNOT gate operations between B (controls) and B' (targets) to copy the syndrome. (iii) Simulate the cooling dynamics in a digitalized way by using the stabilizer pumping [26-29] [see Fig. 5(b)]. (iv) Perform CZ gate operations between A' (controls) and A (targets) to apply

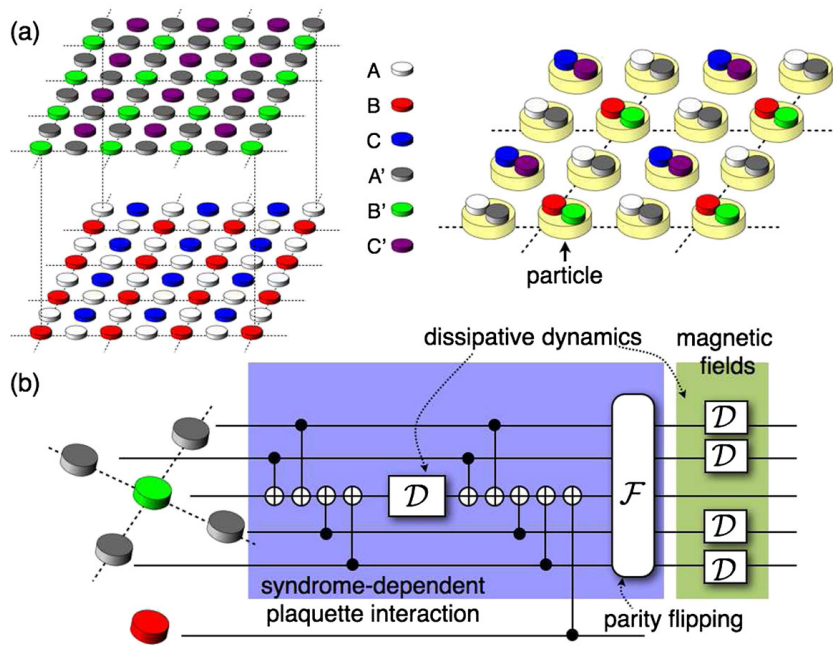

FIG. 5. Physical implementation of MFTP. (a) The double-layer system for MFTP. Each 2D layer consists of three species of particles (left). The different degrees of freedom of the particles on a single 2D layer can also be utilized (right). (b) The stabilizer pumping scheme for a digital simulation of the cooling process (see Appendix B). 


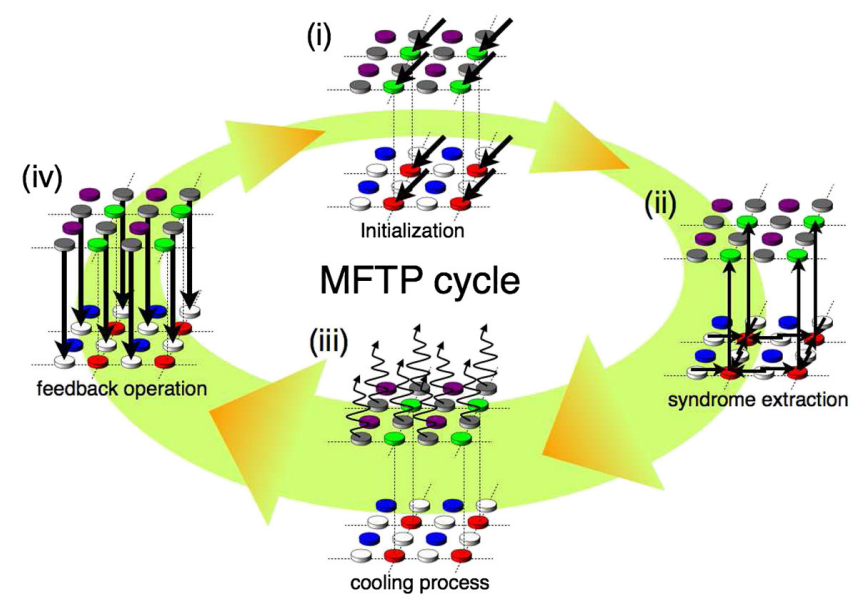

FIG. 6. One MFTP cycle for the $Z$ error correction. (i) The $B$ qubits and B' spins are initialized to extract and copy the error syndrome, respectively. (ii) The error syndrome is extracted from the A qubits to the B qubits. The extracted error syndrome is copied to the B' spins. (iii) By using the copied error syndrome, two-qubit gates, and single-qubit dissipative dynamics, the cooling process under the Hamiltonian $H\left(\left\{b_{v}\right\}\right)$ is simulated in a digitalized way [see Fig. 5(b)]. (iv) The obtained equilibrium configuration, parts of which reveal the location of the $Z$ errors, is put back into the A qubits to correct the $Z$ errors.

the feedback operation. A similar procedure for the $X$ error correction is also performed with a basis change using Hadamard operations. These MFTP cycles for the $X$ and $Z$ error corrections are repeated.

The cooling process in step (iii) is simulated in a digitalized way, which we call digitalized cooling, using stabilizer pumping [3,26-29]. We can realize the syndromedependent plaquette interactions automatically by using the five-body interactions described by

$$
\tilde{H}=-J \sum_{v} Z_{v}^{B^{\prime}} \prod_{i \in E_{v}} Z_{i}^{A^{\prime}}-h \sum_{i} Z_{i}^{A^{\prime}}
$$

since the error syndrome is copied onto the B' spins in step (ii). Note that the classical spins A', B', and C' are denoted as if they are qubits, for simplicity of the notation. By introducing an interaction $H_{\text {int }}$ between the ancilla classical spins and the environment, the time evolution of the total system is given by $V(t) \equiv e^{-i\left(\tilde{H}+H_{\text {int }}\right) t}$. If the coupling strength with the environment is sufficiently small, and the correlation time is sufficiently short, then the time evolution of the system can be regarded as a Markovian decay. Then, the time evolution is divided into short digitalized cooling steps of interval $\tau=t / m$ :

$$
V(t) \simeq\left[e^{-i\left(H_{P}+H_{\text {int }} / 2\right) \tau} e^{-i\left(H_{F}+H_{\text {int }} / 2\right) \tau}\right]^{m},
$$

where $H_{P} \equiv-J \sum_{v} Z_{v}^{B^{\prime}} \prod_{i \in E_{v}} Z_{i}^{A^{\prime}}, H_{F} \equiv-h \sum_{i} Z_{i}^{A^{\prime}}$, and the Trotter-Suzuki expansion [45] is employed. By tracing out the environment, we obtain Markovian digitalized cooling dynamics,

$$
\operatorname{Tr}_{E}\left[V(t) \rho_{A} \otimes \rho_{E} V(t)^{\dagger}\right] \simeq\left[e^{\tau \mathcal{L}_{P}} e^{\tau \mathcal{L}_{F}}\right]^{m} \rho_{A},
$$

where $\rho_{A}$ and $\rho_{E}$ indicate density matrices of the ancilla system and the environment, respectively. $\mathcal{L}_{P}$ and $\mathcal{L}_{F}$ denote the Lindblad superoperators [46] with respect to the Markovian decays under $H_{P}$ and $H_{F}$, respectively. These are realized by using single-qubit dissipative dynamics and two-qubit gates, as shown in Fig. 5 [26-29] (see Appendix B).

We now discuss the requirements of the physical parameters in the experiments. The decoherence rate of the quantum system $\mathrm{A}, \mathrm{B}$, and $\mathrm{C}$ is denoted by $\Gamma$. The strength of the dissipative operation on the classical spins A', B', and C' is denoted by $\gamma \sim\left\|H_{\text {int }}\right\|$. We also define a coupling strength $\kappa$ between the qubits and spins, which limits the gate time to $1 / \kappa$. Furthermore, we utilize a Markov approximation, $J, h \gg \gamma$, and the Trotter-Suzuki expansion imposes $J \gamma \tau^{2} \ll 1$ and $h \gamma \tau^{2} \ll 1$. By setting $J, h \sim 10 \gamma, J \gamma \tau^{2} \sim 10^{-1}$, and $h \gamma \tau^{2} \sim 10^{-1}$, the time interval $\tau$ of each digitalized cooling step becomes $\tau \sim 10^{-1} / \gamma$. According to the numerical simulations at least up to the system size $L=400$, the cooling process is accomplished within $\sim 10^{2}-10^{4}$ Monte Carlo steps, each of which physically takes a relaxation time of $\sim 1 / \gamma$ for local spin flipping. Thus, the cooling time $t_{\text {cool }}$ required for one MFTP cycle is estimated to be $t_{\text {cool }} \sim 10^{2}-10^{4} / \gamma$.

Since the cooling time $t_{\text {cool }}$ is divided into digitalized cooling steps of time interval $\tau \sim 10^{-1} / \gamma$, the number of repetitions is $m=t_{\text {cool }} / \tau \sim 10^{3}-10^{5}$. In each digitalized cooling step, only a few unitary operations are required since most of the unitary gates are commutable and operate simultaneously. Accordingly, in addition to the cooling time $t_{\text {cool }}$, the unitary gate operations take $\sim m / g$. As a result, the time taken by one MFTP cycle is

$$
t_{\text {cycle }}=t_{\text {cool }}+m / \kappa \sim 10^{2} / \gamma+10^{3} / \kappa .
$$

In order for MFTP to work, the physical error probability $p=1-e^{-\Gamma t_{\text {cycle }}}$ for the quantum system has to be as small as $10^{-2}$. (When both $X$ and $Z$ errors are corrected using the same classical layer, the physical error probability effectively doubled.) Hence, we obtain requirements on the physical parameters, $\Gamma / \gamma \lesssim 10^{-4}-10^{-6}$ and $\Gamma / \kappa \lesssim 10^{-5}$. The former and latter conditions indicate that the speeds of dissipation and unitary operations have to be $10^{4}-10^{6}$ and $10^{5}$ times faster than the decoherence time of the quantum system, respectively. If these conditions are satisfied, the effective decay rate per cycle is reduced to $\sim 10^{-8}$ (the physical decoherence rate is reduced 6 orders of magnitude) assuming the scaling for $L=400$.

Here, we discuss the feasibility of the above conditions in the case of a solid-state system with nuclear and electron 
spins for quantum and classical systems, respectively. State-of-the-art chemistry and nanotechnology research has demonstrated the possibility of engineering materials with nuclear and electron spins arranged on a lattice. In solids such as diamond, the $T_{2}$ relaxation time of nuclear spins exceeds $1 \mathrm{sec}$ even if it is located near an electron spin [47]. The electron spins can be initialized within $1 / \gamma \sim 100 \mathrm{nsec}$ if they have appropriate optical transitions. The hyperfine interaction between electron and nuclear spins and the dipolar interaction between electron spins for two-qubit gates are both typically on the order of $\kappa \sim 1-100 \mathrm{MHz}$. These parameters fulfill the above conditions. In such a case, $t_{\text {cycle }}=1 \mathrm{msec}$, and the lifetime of quantum information under MFTP with $L=400$ becomes $10^{5}$ sec, i.e., more than 1 day.

Other degrees of freedom in natural or artificial atoms, for example, such as the motional and internal degrees of freedom in trapped atoms [26-29] or superconducting flux qubits coupled with microwave cavities [16,17], can also be utilized.

\section{CONCLUSION AND DISCUSSION}

We propose MFTP, which is a novel way to protect quantum information from decoherence using dissipative operations without parallel projective measurements or selective addressing. We show that local and translationally invariant physical operations without any selective addressing and measurements achieve topological protection of quantum information against errors on the code state. A fully fault-tolerant analysis should be made, further taking into account those imperfections in the syndrome extraction and the engineered dissipative dynamics. To this end, the classical ancilla system, instead of using a fully quantum system, of a finite temperature without necessity of phase coherence would have an advantage with respect to its robustness. Moreover, for fault-tolerant quantum computation, we have to engineer the boundaries of planer surface codes in order to accommodate many logical qubits and to perform logical gates between them.

Recently, quantum memory (surface codes) coupled with another quantum or classical system, such as classical fields, spins, or a bosonic reservoir, has been investigated extensively to increase the lifetime of quantum information $[43,48,49]$. Among them, the automaton decoder [48], where anyonic excitations (boundaries of error chains) are annihilated by an attractive force given by classical potential fields made by the excitations themselves, shares the idea with our proposal. The automaton decoder would also be made measurement-free if there was a feasible way to implement the classical fields and their update rules.

The dissipative dynamics has recently been well studied in quantum simulation. Actually, the key ingredients of MFTP have already been demonstrated in quantum simulation [27-29]. The proposed model, an active use of the engineered dissipative dynamics for topological protection, establishes a promising means of progressing from quantum simulation to fault-tolerant quantum computing.

\section{ACKNOWLEDGMENTS}

The authors thank S. Nishida, Y. Morita, H. Tasaki, and B. Yoshida for valuable discussions. This work was supported by the Funding Program for World-Leading Innovative Research and Development on Science and Technology (FIRST), MEXT Grant-in-Aid for Scientific Research on Innovative Areas 20104003 and 21102008, the MEXT Global COE Program, and JSPS Grant-in-Aid for Research Activity Start-up 25887034.

\section{APPENDIX A: ANALYSIS OF THE LOGICAL ERROR PROBABILITY}

The energy penalty for the boundary mismatching is $4 J$, since they are always created as a pair of excitations with respect to plaquette interactions. On the other hand, a connected chain of -1 's of a length $r$ costs an energy $2 h r$ due to the magnetic fields. Thus, the pair of two wrong-sign plaquettes is connected in a typical case when the distance between two wrong-sign plaquettes is shorter than $r_{\text {cor }} \equiv 4 J /(2 h)$, which gives a typical length of the system (more precisely, the maximum correlation length at zero temperature). If errors occur contiguously, and the distance between two wrong-sign plaquettes becomes larger than $r_{\text {cor }}$, then the boundary mismatching is energetically favored due to the energy penalty of magnetic fields. This means that the spins of the classical system search pairs of wrong-sign plaquettes inside an area whose vertical and horizontal lengths are typically given by $r_{\text {cor }}$.

In the following, we bound the logical error probability after the feedback operation through a two-step argument. First, we consider a necessary condition: the energy landscape of the classical system $H\left(\left\{b_{v}\right\}\right)$ is given appropriately such that the spin configuration at zero temperature can correct the error appropriately. This is not sufficient for our purpose, since we utilize a spin configuration at finite temperature. Thus, second, we bound the logical error probability for the given appropriate energy landscape taking into account the finite temperature effect.

For a given error chain $C_{e}$, we define a minimum-path chain $C_{m}$, which connects pairs of wrong-sign plaquettes with a shortest path (i.e., the solution of MWPM). When errors are dense, the error and minimum-path chains $C_{e}+$ $C_{m}$ would constitute a connected chain of a length longer than $r_{\text {cor }}$. In such a case, the error correction fails even if we have the spin configuration at zero temperature, resulting in an inappropriate energy landscape. To calculate such a failure probability $p_{\text {error }}$, we consider an area whose vertical and horizontal lengths are given by $r_{\text {cor }}$. The probability that the error and minimum-path chains $C_{e}+C_{m}$ constitute a connected chain of length $l \geq r_{\text {cor }}$ can be calculated by following the method provided in Ref. [31]: 


$$
\begin{aligned}
p_{\text {error }} & <\sum_{l \geq r_{\text {cor }}} C(l) \sum_{k>l / 2}^{l}\left(\begin{array}{l}
l \\
k
\end{array}\right) p^{k}(1-p)^{l-k} \\
& <\sum_{l \geq r} C(l) 2^{l} p^{l / 2}
\end{aligned}
$$

where $C(l)$ is the number of self-avoiding walks of length $l$ inside this area, which is bounded as $C(l)<3 \times 4^{l-1}$. Since we can begin such a connected chain of length $l \geq$ $r_{\text {cor }}$ at any one of $L^{2}$ lattice sites, the total failure probability is at most $1-\left(1-p_{\text {error }}\right)^{L^{2}} \simeq L^{2} p_{\text {error }}$. [In STQEC with MWPM, $r_{\text {error }}$ is replaced with the system size $L$. This reproduces the calculation made in Ref. [31], leading to the threshold value independent of the system size. The fact that $r_{\text {error }}=O(L)$ implies that communication over the whole system is employed in the classical processing for MWPM, which requires a time $O(L)$ in the presence of parallelism. By using an elaborated renormalization algorithm, we can reduce the time complexity for this communication to $O(\log L)$ [50], where nonlocal operations, which are prohibited in the present setup, are still required. See also Table I.]

Since we utilize a spin configuration at finite temperature, we further take into account excitations that originate from the finite temperature effect to calculate the logical error rate. There are two types of excitations: (i) excitations without boundary mismatches and (ii) excitations with boundary mismatches. In the former case (i), while the ground-state configuration corresponds to an appropriate location of the errors, excitations with respect to the magnetic fields help to form a connected chain of length longer than $r_{\text {cor }}$. Such a probability is calculated as

$$
\begin{aligned}
p_{\mathrm{ex}} & =\sum_{l \geq r_{\mathrm{cor}}} C(l) \sum_{k=0}^{l / 2}\left(\begin{array}{l}
l \\
k
\end{array}\right) p^{k}(1-p)^{l-k} e^{-2 \beta h(l-2 k)} p_{\mathrm{gs}} \\
& <\sum_{l \geq r_{\mathrm{cor}}} C(l) \sum_{k=0}^{l / 2}\left(\begin{array}{l}
l \\
k
\end{array}\right)\left[e^{-2 \beta h}(1-p)\right]^{l-k}\left(p / e^{-2 \beta h}\right)^{k} \\
& <\sum_{l \geq r_{\mathrm{cor}}} C(l) 2^{l} p^{l / 2},
\end{aligned}
$$

where $p_{\mathrm{gs}} \geq 1$ is the population of the ground state, and the temperature is chosen to be $e^{-2 \beta h}=p /(1-p)$. The condition $e^{-2 \beta h}=p /(1-p)$, the so-called Nishimori line [44], indicates that the thermal fluctuation with respect to the magnetic field and the physical error probability are balanced. As a result, we can obtain the same scaling of the failure probabilities $p_{\text {error }}$ and $p_{\mathrm{ex}}$ to the leading order in the presence of the thermal fluctuation below the Nishimori line. Similarly to the previous case, the connected chain of length $l \geq r_{\text {cor }}$ can begin at any one of $L^{2}$ lattice sites, and hence, the total failure probability in this case is at most $L^{2} p_{\mathrm{ex}}$.

Finally, we consider the latter case (ii). Since the number of connected errors in this area is at most $r_{\text {cor }} / 2$, the boundary mismatching costs at least $4 J-2 h\left(r_{\text {cor }} / 2\right)$ energy penalty. Thus, the probability of the excitation causing the boundary mismatching can be given by

$$
p_{\mathrm{bm}}=e^{-4 \beta J+2 \beta h\left(r_{\mathrm{cor}} / 2\right)} p_{\mathrm{gs}}<e^{-\beta h r_{\mathrm{cor}}} .
$$

Such a boundary mismatching can occur at any one of $L^{2}$ plaquettes, and the total failure probability in this case

\begin{tabular}{|c|c|c|c|c|c|c|}
\hline Model & Type & $\begin{array}{l}\text { Coupling } \\
\text { strength }\end{array}$ & $\begin{array}{l}\text { Memory } \\
\text { time }\end{array}$ & $\begin{array}{l}\text { Decoding } \\
\text { time }\end{array}$ & $\begin{array}{l}\text { Type of operations } \\
\text { employed (in 3D) }\end{array}$ & Dynamics \\
\hline Our proposal with $J=O(h \log L)$ & Active & $O(\log L)$ & $\operatorname{poly}(L)$ & poly $\log (L)$ & $\begin{array}{l}\text { Local and translationally } \\
\text { invariant }\end{array}$ & $\begin{array}{l}\text { Nonequilibrium } \\
\text { driven by feedback }\end{array}$ \\
\hline Our proposal with $J=O(h L)$ & Active & $O(L)$ & $\exp (L)$ & $\operatorname{poly}(L)$ & $\begin{array}{l}\text { Local and translationally } \\
\text { invariant }\end{array}$ & $\begin{array}{l}\text { Nonequilibrium } \\
\text { driven by feedback }\end{array}$ \\
\hline $\begin{array}{l}\text { Surface code in } 4 \mathrm{D} \text { without } \\
\text { error correction [31] }\end{array}$ & Passive & Constant & $\exp (L)$ & No & $\begin{array}{l}\text { Nonlocal translationally } \\
\text { invariant }\end{array}$ & Thermal equilibrium \\
\hline $\begin{array}{l}\text { Surface code in 2D without } \\
\text { error correction [51] }\end{array}$ & Passive & Constant & Constant & No & $\begin{array}{l}\text { Local and translationally } \\
\text { invariant }\end{array}$ & Thermal equilibrium \\
\hline Toric-boson model [43] & Passive & $\operatorname{poly}(L)$ & $\operatorname{poly}(L)$ & No & $\begin{array}{l}\text { Local and translationally } \\
\text { invariant }\end{array}$ & Thermal equilibrium \\
\hline $\begin{array}{l}\text { Surface code in 2D with } \\
\text { MWPM [31] }\end{array}$ & Active & Not defined & $\exp (L)$ & $\operatorname{poly}(L)$ & $\begin{array}{l}\text { Nonlocal and no } \\
\text { translationally invariant }\end{array}$ & $\begin{array}{l}\text { Nonequilibrium } \\
\text { driven by feedback }\end{array}$ \\
\hline $\begin{array}{l}\text { Surface code in } 2 \mathrm{D} \text { with a } \\
\text { renormalization decoder [50] }\end{array}$ & Active & Not defined & $\exp (L)$ & polylog $(L)$ & $\begin{array}{l}\text { Nonlocal but having } \\
\text { self-similarity }\end{array}$ & $\begin{array}{l}\text { Nonequilibrium } \\
\text { driven by feedback }\end{array}$ \\
\hline
\end{tabular}
amounts $L^{2} p_{\mathrm{bm}}$.

TABLE I. A comparison of the performances of the related topological protection schemes based on the surface codes. The types of protections, active and passive, indicate the noise models and how to suppress errors. The active type mainly assumes probabilistic errors on the surface code, which are corrected by a feedback process. The passive type assumes thermal excitations on the surface code (the Toric code model), which are suppressed by an energy penalty coupling with an ancilla system or a higher dimensionality. 
Summing all of these failure probabilities, the logical error probability is given by

$$
\begin{aligned}
p_{\text {logical }} & =L^{2} p_{\text {error }}+L^{2} p_{\text {ex }}+L^{2} p_{\mathrm{bm}} \\
& <L^{2}\left(\sum_{l \geq r_{\text {cor }}} \frac{8}{3} 6^{l} p^{l / 2}+[p /(1-p)]^{r_{\text {cor }} / 2}\right) .
\end{aligned}
$$

Suppose the typical length $r_{\text {cor }}$ is chosen to be $r_{\text {cor }}=$ $\alpha \log L$ with a constant $\alpha$. The failure probability converges to zero in the limit of a large $L$ if $p /(1-p) \leq e^{-4 / \alpha} / 36$. For example, with $\alpha=1$ and 2 , this condition reads the threshold value $p_{\text {th }} \simeq 5.1 \times 10^{-4}$ and $3.7 \times 10^{-3}$, respectively. We note that the above calculation is far from tight, since $C(l)$ is overestimated substantially. Furthermore, in many cases the boundary mismatches do not cause a fatal failure, since the remaining errors would be corrected in the following steps. Thus, the true threshold value is expected to be much higher, as shown in the numerical simulation.

The relaxation time is an important factor for the proposed scheme, since the physical error probability $p$ is increased exponentially during the delay of the feedback. The relaxation time is typically given as a polynomial function of the correlation length of the system. [In a critical system, the typical time scale of the system $\tau_{\text {crit }}$ and the correlation length $r_{\text {crit }}$ are related by $\tau_{\text {crit }}=r_{\text {crit }}^{z}$, with $z$ being the dynamical critical exponent. The present classical system $H\left(\left\{b_{v}\right\}\right)$ is not critical, and the relaxation time is expected to be a lower order polynomial function than $r_{\text {cor }}^{z}$.] In the present case, the typical length of the system depends on the system size only logarithmically, $r_{\text {cor }}=\alpha \log L$. Thus, the relaxation time required to obtain a thermal equilibrium state or a metastable state enough to execute error correction is expected to be a polylogarithmic function of the system size $L$.

We numerically confirm that the classical system rapidly decays to the thermal equilibrium state when the inverse temperature is chosen to be $e^{-4 \beta J} \sim 10^{-3}$. Specifically, the equilibration is accomplished within several hundred Monte Carlo steps at least up to the system size $L=400$. This is because metastable states relatively easily climb up the energy barrier and find the pairs at such a temperature, as shown in Fig. 7. Then the temperature of the classical system is further reduced to the Nishimori temperature, where the unpaired spin chains are also shrunk due to the energy penalty $e^{-\beta h}$. In numerical simulations, with less than $10^{4}$ Monte Carlo steps, we can prepare the classical ancilla system of the system size at least $L=400$. At least up to $L=400$, the relaxation time scales like a polylogarithmic function of the system size $L$. With a small probability, the classical ancilla system is still a metastable state due to the energy barrier $4 \beta J$ at the low temperature. However, error correction using such a metastable state does not spoil error correction, since the length of unpaired spin chains is suppressed due to the energy penalty at low

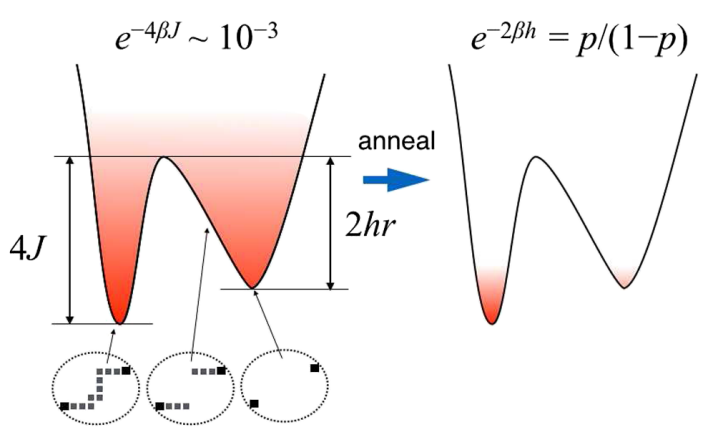

FIG. 7. The energy landscape of the ancilla system. At a high temperature, such as $e^{-4 \beta J}=10^{-3}$, the system decays to the thermal equilibrium state rapidly. Then the temperature is further reduced to the Nishimori temperature. The relaxation time scales polylogrithmically in the size $L$ of the system at least up to $L=400$, where at most $10^{4}$ Monte Calro steps are enough for equilibration with unsophisticated schedulings of annealing.

temperature, as shown in Fig. 7. Then, such a remaining error may be corrected in the next round.

Based on these arguments, we expect that the delay time $\tau$ for the feedback scales like $\tau \sim$ poly $\log (L)$. The threshold for the decoherence rate of the quantum system scales like $\Gamma_{\text {th }} \sim 1 /$ poly $\log (L)$. In practice, it should be better to accomplish an error correction procedure as fast as possible rather than waiting a slow convergence to the thermal equilibrium state. The optimization of the annealing schedule of the classical ancilla system is an intriguing future issue.

Finally, we consider a large $J$ limit, where $J$ is scaled as $O(h L)$. In such a case, $r_{\text {cor }}$ scales like $O(L)$. Thus, the discrete-dissipative feedback at the Nishimori temperature provides an optimal decoding, which is slightly better than the decoding with MWPM [52,53]. An exponential suppression of the logical error can also be achieved under this scaling. However, if the coupling strength scales like $J \sim O(h L)$, the relaxation time (Monte Carlo steps) would also scale polynomially in the size of the system. It is still open whether or not the fast scheduling of classical dissipative dynamics, possibly using metastable states, can be managed to suppress the logical error exponentially. We summarize the performance of the proposed scheme compared with other related models, including decoding methods based on the explicit parallel measurements and the classical processing in Table I.

\section{APPENDIX B: DIGITAL SIMULATION OF THE COOLING PROCESS}

The Lindblad superoperators $\mathcal{L}_{P}$ and $\mathcal{L}_{F}$ for Markovian decays under the Hamiltonian $H_{P}$ and $H_{F}$ are given by

$$
\begin{aligned}
\mathcal{L}_{P} \rho= & -\gamma_{-} \sum_{v \in B^{\prime}}\left(\hat{\eta}_{v}^{\dagger} \hat{\eta}_{v} \rho+\rho \hat{\eta}_{v}^{\dagger} \hat{\eta}_{v}-2 \hat{\eta}_{v} \rho \hat{\eta}_{v}^{\dagger}\right) \\
& -\gamma_{+} \sum_{v \in B^{\prime}}\left(\hat{\eta}_{v} \hat{\eta}_{v}^{\dagger} \rho+\rho \hat{\eta}_{v} \hat{\eta}_{v}^{\dagger}-2 \hat{\eta}_{v}^{\dagger} \rho \hat{\eta}_{v}\right),
\end{aligned}
$$




$$
\begin{aligned}
\mathcal{L}_{F} \rho= & -\gamma_{-}^{\prime} \sum_{i \in A^{\prime}}\left(\hat{\sigma}_{i}^{+} \hat{\sigma}_{i}^{-} \rho+\rho \hat{\sigma}_{i}^{+} \hat{\sigma}_{i}^{-}-2 \hat{\sigma}_{i}^{-} \rho \hat{\sigma}_{i}^{+}\right) \\
& -\gamma_{+}^{\prime} \sum_{i \in A^{\prime}}\left(\hat{\sigma}_{i}^{-} \hat{\sigma}_{i}^{+} \rho+\rho \hat{\sigma}_{i}^{-} \hat{\sigma}_{i}^{+}-2 \hat{\sigma}_{i}^{+} \rho \hat{\sigma}_{i}^{-}\right) .
\end{aligned}
$$

The operator $\hat{\eta}_{v}=X_{i \in E_{v}}^{A^{\prime}}\left(I-Z_{v}^{B^{\prime}} \prod_{i \in E_{v}} Z_{i}^{A^{\prime}}\right) / 2$, where $i \in$ $E_{v}$ means that one of the edges in $E_{v}$ is chosen randomly, lowers the energy with respect to $-J Z_{v}^{B^{\prime}} \prod_{i \in E_{v}} Z_{i}^{A^{\prime}}$. The operators $\hat{\sigma}_{i}^{+}=|1\rangle\left\langle\left. 0\right|_{i}\right.$ and $\left.\hat{\sigma}_{i}^{-}=\mid 0\right\rangle\left\langle\left. 1\right|_{i}\right.$ are the raising and lowering operators for the $i$ th qubit, respectively. The ratio of the decay and the excitation rates is given in terms of the inverse temperature of the environment and the energy gaps $J$ and $h$ of the system as follows: $\gamma_{-} / \gamma_{+}=e^{-2 \beta J} /$ $\left(1+e^{-2 \beta J}\right)$ and $\gamma_{-}^{\prime} / \gamma_{+}^{\prime}=e^{-2 \beta h} /\left(1+e^{-2 \beta h}\right)$. The singlequbit dissipative dynamics $\mathcal{L}_{F}$ is implemented using a $T_{1}$ relaxation or incoherent pumping.

For the realization of $\mathcal{L}_{P}$ through single-qubit dissipative dynamics and two-qubit gate operations, we introduce another lowering operator, $\hat{\xi}=X_{v}^{B^{\prime}}\left(I-Z_{v}^{B^{\prime}} \prod_{i \in E_{v}} Z_{i}^{A^{\prime}}\right) / 2$. If the parity of $Z_{v}^{B^{\prime}} \prod_{i \in E_{v}} Z_{i}^{A^{\prime}}$ is odd, this operator flips the spin located at the vertex $v \in B^{\prime}$, which is the copy of the syndrome $b_{v}$. Our goal is reducing the energy of the classical system consisting of the A' spins by appropriately flipping the parity of $\prod_{i \in E_{v}} Z^{A^{\prime}}$ as done by $\hat{\eta}$. To this end, if the copy of the syndrome is flipped, we have to flip one of the ancilla spins $i \in E_{v}$. To establish whether the copy of the syndrome is flipped or not, we apply a CNOT gate between the B qubits (controls) to the B' spins (targets). Since the B' spins are copies of the B qubits, this CNOT gate reveals whether the B' spins B' are flipped. If a B' spin is not flipped, the state is +1 after the application of the CNOT gate. If a B' spin is flipped, the state is -1 after the CNOT gate. Consequently, we can change the parity of the A' spins on the corresponding plaquette with random CNOT gates $\mathcal{F}$ between the B' spins (controls) and A' spins (targets),

$$
\mathcal{F} \rho=\frac{1}{4} \sum_{i \in E_{v}} \Lambda(X)_{v, i} \rho \Lambda(X)_{v, i},
$$

where $\Lambda(X)_{v, i}$ is the CNOT gate acting on the $v$ th and $i$ th particles as the control and target, respectively. To change the parity with unitary operations, the random CNOT gates can be replaced with the controlled-exp $\left[-i(\pi / 4) X^{A^{\prime}}\right]$ operation, for example. In this case, the parity is not changed with unit probability, and hence, it may slow down the cooling dynamics. The CNOT gate from the B qubits (controls) to the B' spins (targets) and the parity flipping operation $\mathcal{F}$ are denoted together by $\tilde{\mathcal{F}}$. Followed by the operation $\tilde{\mathcal{F}}$, the effective action of the $\hat{\xi}$ is equivalent to $\hat{\eta}$.

Finally, we describe the dissipative operation under $\hat{\xi}$, whose Lindblad superoperator is denoted by $\tilde{\mathcal{L}}_{P}$. The lowering operator $\hat{\eta}$ is transformed by $U \equiv \prod_{i \in E_{v}} \Lambda(X)_{i, v}$ [see Fig. 5(b)] to

$$
\hat{\xi}_{v}=U X_{v}\left(I-Z_{v} \prod_{i \in E_{v}} Z_{i}\right) U=X_{v}\left(I-Z_{v}\right) / 2=\sigma_{v}^{-},
$$

which is the single-qubit lowering operator for the spins and hence implemented using $T_{1}$ relaxation or incoherent pumping. Thus, by combining the operation $\tilde{\mathcal{F}}$ and $\mathcal{U}$, the dissipative operation $\mathcal{L}_{P}$ can be realized as

$$
e^{\tau \mathcal{L}_{P}} \rho=\tilde{\mathcal{F}} \mathcal{U} e^{\tau \tilde{\mathcal{L}}_{P}} \rho
$$

where $\mathcal{U} \rho \equiv U \rho U$.

[1] P. W. Shor, Scheme for Reducing Decoherence in Quantum Computer Memory, Phys. Rev. A 52, R2493 (1995).

[2] D. P. DiVincenzo and P.W. Shor, Fault-Tolerant Error Correction with Efficient Quantum Codes, Phys. Rev. Lett. 77, 3260 (1996).

[3] B. M. Terhal, Quantum Error Correction for Quantum Memories, arXiv:1302.3428.

[4] A. Yu. Kitaev, Quantum Computations: Algorithms and Error Correction, Russ. Math. Surv. 52, 1191 (1997).

[5] J. Preskill, Reliable Quantum Computers, Proc. R. Soc. A 454, 385 (1998).

[6] E. Knill, R. Laflamme, and W. H. Zurek, Resilient Quantum Computation: Error Models and Thresholds, Proc. R. Soc. A 454, 365 (1998); Resilient Quantum Computation, Science 279, 342 (1998).

[7] D. Aharonov and M. Ben-Or, in Proceedings of the 29th Annual ACM Symposium on the Theory of Computation (ACM Press, New York, 1998), p. 176.

[8] A. M. Steane, Efficient Fault-Tolerant Quantum Computing, Nature (London) 399, 124 (1999).

[9] E. Knill, Quantum Computing with Realistically Noisy Devices, Nature (London) 434, 39 (2005).

[10] R. Raussendorf, J. Harrington, and K. Goyal, A FaultTolerant One-Way Quantum Computer, Ann. Phys. (Amsterdam) 321, 2242 (2006).

[11] R. Raussendorf, J. Harrington, and K. Goyal, Topological Fault-Tolerance in Cluster State Quantum Computation, New J. Phys. 9, 199 (2007).

[12] R. Raussendorf and J. Harrington, Fault-Tolerant Quantum Computation with High Threshold in Two Dimensions, Phys. Rev. Lett. 98, 190504 (2007).

[13] K. Fujii and K. Yamamoto, Cluster-Based Architecture for Fault-Tolerant Quantum Computation, Phys. Rev. A 81, 042324 (2010).

[14] K. Fujii and K. Yamamoto, Topological One-Way Quantum Computation on Verified Logical Cluster States, Phys. Rev. A 82, 060301(R) (2010).

[15] N. C. Jones, R. Van Meter, A. G. Fowler, P. L. McMahon, J. Kim, T. D. Ladd, and Y. Yamamoto, Layered Architecture for Quantum Computing, Phys. Rev. X 2, 031007 (2012).

[16] A. G. Fowler, M. Mariantoni, J. M. Martinis, and A. N. Cleland, Surface Codes: Towards Practical Large-Scale Quantum Computation, Phys. Rev. A 86, 032324 (2012). 
[17] J. Ghosh, A. G. Fowler, and M. R. Geller, Surface Code with Decoherence: An Analysis of Three Superconducting Architectures, Phys. Rev. A 86, 062318 (2012).

[18] J. I. Cirac, A. K. Ekert, S. F. Huelga, and C. Macchiavello, Distributed Quantum Computation over Noisy Channels, Phys. Rev. A 59, 4249 (1999).

[19] W. Dür and H.-J. Briegel, Quantum Repeaters: The Role of Imperfect Local Operations in Quantum Communication, Phys. Rev. Lett. 90, 067901 (2003).

[20] L. Jian, J. M. Taylor, A. S. Sørensen, and M. D. Lukin, Distributed Quantum Computation Based on Small Quantum Registers, Phys. Rev. A 76, 062323 (2007).

[21] Y. Li, S. D. Barrett, T. M. Stace, and S. C. Benjamin, Fault Tolerant Quantum Computation with Nondeterministic Gates, Phys. Rev. Lett. 105, 250502 (2010).

[22] K. Fujii and Y. Tokunaga, Fault-Tolerant Topological OneWay Quantum Computation with Probabilistic Two-Qubit Gates, Phys. Rev. Lett. 105, 250503 (2010).

[23] K. Fujii, T. Yamamoto, M. Koashi, and N. Imoto, A Distributed Architecture for Scalable Quantum Computation with Realistically Noisy Devices, arXiv:1202.6588.

[24] Y. Li and S. C. Benjamin, High Threshold Distributed Quantum Computing with Three-Qubit Nodes, New J. Phys. 14, 093008 (2012).

[25] C. Monroe, R. Raussendorf, A. Ruthven, K. R. Brown, P. Maunz, L.-M. Duan, and J. Kim, Large-Scale Modular Quantum-Computer Architecture with Atomic Memory and Photonic Interconnects, Phys. Rev. A 89, 022317 (2014).

[26] M. Müller, K. Hammerer, Y. L. Zhou, C. F. Roos, and P. Zoller, Simulating Open Quantum Systems: From Many-Body Interactions to Stabilizer Pumping, New J. Phys. 13, 085007 (2011).

[27] H. Weimer, M. Müller, I. Lesanovsky, P. Zoller, and H. P. Büchler, A Rydberg Quantum Simulator, Nat. Phys. 6, 382 (2010).

[28] J. T. Barreiro, M. Müller, P. Schindler, D. Nigg, T. Monz, M. Chwalla, M. Hennrich, C. F. Roos, P. Zoller, and R. Blatt, An Open-System Quantum Simulator with Trapped Ions, Nature (London) 470, 486 (2011)

[29] B. P. Lanyon, C. Hempel, D. Nigg, M. Müller, R. Gerritsma, F. Zähringer, P. Schindler, J. T. Barreiro, M. Rambach, G. Kirchmair, M. Hennrich, P. Zoller, R. Blatt, and C. F. Roos, Universal Digital Quantum Simulation with Trapped Ions, Science 334, 57 (2011).

[30] A. Yu. Kitaev, Fault-Tolerant Quantum Computation by Anyons, Ann. Phys. (Amsterdam) 303, 2 (2003).

[31] E. Dennis, A. Kitaev, A. Landahl, and J. Preskill, Topological Quantum Memory, J. Math. Phys. (N.Y.) 43, 4452 (2002).

[32] H. Bombin and M. A. Martin-Delgado, Topological Quantum Distillation, Phys. Rev. Lett. 97, 180501 (2006).

[33] J. Edmonds, Paths, Trees, and Flowers, Can. J. Math. 17, 449 (1965).

[34] J. Fitzsimons and J. Twamley, Quantum Fault Tolerance in Systems with Restricted Control, Electron. Notes Theor. Comput. Sci. 258, 35 (2009).
[35] G. A. Paz-Silva, G. K. Brennen, and J. Twamley, Fault Tolerance with Noisy and Slow Measurements and Preparation, New J. Phys. 13, 013011 (2011).

[36] J. W. Harrington, Ph.D. thesis, California Institute of Technology, 1997.

[37] B. Kraus, H. P. Büchler, S. Diehl, A. Kantian, A. Micheli, and P. Zoller, Preparation of Entangled States by Quantum Markov Processes, Phys. Rev. A 78, 042307 (2008).

[38] F. Verstraete, M. M. Wolf, and J. I. Cirac, Quantum Computation and Quantum-State Engineering Driven by Dissipation, Nat. Phys. 5, 633 (2009).

[39] K. G. H. Vollbrecht, C. A. Muschik, and J. I. Cirac, Entanglement Distillation by Dissipation and Continuous Quantum Repeaters, Phys. Rev. Lett. 107, 120502 (2011).

[40] F. Pastawski, L. Clemente, and J. I. Cirac, Quantum Memories Based on Engineered Dissipation, Phys. Rev. A 83, 012304 (2011).

[41] S. Bravyi and B. Terhal, A No-Go Theorem for a Two-Dimensional Self-Correcting Quantum Memory Based on Stabilizer Codes, New J. Phys. 11, 043029 (2009).

[42] B. Yoshida, Feasibility of Self-Correcting Quantum Memory and Thermal Stability of Topological Order, Ann. Phys. (Amsterdam) 326, 2566 (2011).

[43] A. Hamma, C. Castelnovo, and C. Chamon, Toric-Boson Model: Toward a Topological Quantum Memory at Finite Temperature, Phys. Rev. B 79, 245122 (2009).

[44] H. Nishimori, Internal Energy, Specific Heat and Correlation Function of the Bond-Random Ising Model, Prog. Theor. Phys. 66, 1169 (1981).

[45] M. Suzuki, General Theory of Higher-Order Decomposition of Exponential Operators and Symplectic Integrators, Phys. Lett. A 165, 387 (1992).

[46] G. Lindblad, On the Generators of Quantum Dynamical Semigroups, Commun. Math. Phys. 48, 119 (1976).

[47] P. C. Maurer, G. Kucsko, C. Latta, L. Jiang, N. Y. Yao, S. D. Bennett, F. Pastawski, D. Hunger, N. Chisholm, M. Markham, D. J. Twitchen, J. I. Cirac, and M. D. Lukin, Room-Temperature Quantum Bit Memory Exceeding One Second, Science, 336, 1283 (2012).

[48] M. Herold, E. T. Campbell, J. Eisert, and M. J. Kastoryano, Cellular-Automaton Decoders for Topological Quantum Memories, arXiv:1406.2338.

[49] E. Kapit, J. T. Chalker, and S. H. Simon, Passive Correction of Quantum Logical Errors in a Driven, Dissipative System: A Blueprint for an Analog Quantum Code Fabric, arXiv:1408.0959.

[50] G. Duclos-Cianci and D. Poulin, Fast Decoders for Topological Quantum Codes, Phys. Rev. Lett. 104, 050504 (2010).

[51] R. Alicki, M. Fannes, and M. Horodecki, On Thermalization in Kitaev's 2D Model, J. Phys. A 42, 065303 (2009).

[52] F. Merz and J. T. Chalker, Two-Dimensional Random-Bond Ising Model, Free Fermions, and the Network Model, Phys. Rev. B 65, 054425 (2002).

[53] M. Ohzeki, Locations of Multicritical Points for Spin Glasses on Regular Lattices, Phys. Rev. E 79, 021129 (2009). 